\title{
Enhanced Threshold Sensitive Stable Election Protocol using Fuzzy Logic for Wireless Sensor Networks
}

\author{
Shailie Rai \\ M.Tech Scholar \\ Department of Electronics \\ and Communication \\ Babu Banarasi Das \\ University Lucknow, India
}

\author{
Pallavi Gupta \\ Assistant Professor \\ Department of Electronics and \\ Communication \\ Babu Banarasi Das University \\ Lucknow, India
}

\begin{abstract}
In this paper, an Enhanced Threshold Sensitive Stable Election Protocol using Fuzzy Logic (ETSSEPFL) is proposed to prolong the lifespan of heterogeneous wireless sensor networks (HWSNs). It improves existing SEP and TSEP protocols by using a fuzzy logic expert system which intelligently assigns cluster heads (CHs) which lead to energy efficient HWSN. The focus is on two linguistic variables for the design of a fuzzy expert system, which include residual energy and distance to a base station (BS). The simulation results of this research are compared with SEP and TSEP protocols to evaluate the performance of the proposed routing protocol. The evaluation concludes that the proposed routing protocol is better in prolonging network lifetime, increasing stability period and throughput. ETSSEPFL builds a more stable routing environment as compared to SEP and TSEP.
\end{abstract}

\section{Keywords}

Wireless sensor networks, clustering, threshold sensitive stable election protocol, fuzzy logic.

\section{INTRODUCTION}

Wireless sensor networks (WSN) consists of several routing protocols that have been proposed for different field of applications. WSN have a dynamic environment, limited power and memory so to build a simple, scalable and efficient routing protocol become one of the most challenging tasks. Various applications of sensor networks are tracking, monitoring of habitat, battlefield surveillance, home automation and many others [1,2,3]. WSNs have limited energy as they are battery operated; recharge of the battery is not supported. Thus the development of techniques for extending the battery lifetime is essential. Several routing protocols have been proposed for different field of applications so as to achieve energy-efficiency and enhanced network lifetime.

An efficient solution to prolong the network lifetime is clustering of sensor nodes. It offers several advantages such as load balancing, scalability, reduction in collisions during intra-cluster communication [4]. There are two categories of cluster head selection schemes. In the homogeneous schemes, all the sensor nodes of the network are equipped with the same amount of energy such as LEACH[5], HEED[6], CHEF[7].

However, the performance of homogeneous schemes is poor for heterogeneous networks as the nodes with low energy level could have a high probability of election than the nodes with high energy level. In the heterogeneous schemes, all sensor nodes have a different amount of energy as each node is assigned with various task [8],[9] such as SEP[10], DEEC[11].

In this paper, we propose and analyze a novel cluster head selection scheme based on Fuzzy Inference System (FIS) for heterogeneous wireless sensor networks (HWSNs) which is named as ETSSEPFL. In ETSSEPFL, the node's qualification for being a $\mathrm{CH}$ is evaluated as per the residual energy of a node and distance to a base station (BS). Based on these factors, each node applies the fuzzy rules to determine whether to become a cluster head in each round. The nodes that have high residual energy and are closer to base station have the high probability of being a cluster head.

The remainder of this paper is organized as follows. In Section 2 related work is discussed. In Section 3, the Radio Energy Dissipation Model is described. Section 4 provides a brief description of Fuzzy approach. In Section 5, ETSSEPFL mechanism is discussed in detail. Section 6 provides a comparative analysis of ETSSEPFL with SEP and TSEP protocols. Finally, Section 7 concludes the paper.

\section{RELATED WORK}

\subsection{LEACH}

Heinzelman et al. proposed Low Energy Adaptive Clustering Hierarchy (LEACH) protocol [5]. LEACH was the first hierarchical and reactive routing protocol for the wireless sensor networks. It is a protocol, which performs well in homogeneous networks, where all the sensor nodes have an identical amount of energy. In this protocol sensor nodes are divided into some clusters then cluster heads are elected from these clusters that aggregate data from the sensor nodes and finally transfer the data to the base station (BS). Hence, in the network only cluster head transmits the data to the base station instead of all the sensor nodes thus, energy consumption is low. In this protocol, it is assumed that the base station or the sink is fixed. The crux of this protocol is to form clusters of sensor nodes. Energy consumption is uniform as it randomly selects a cluster head for each cluster. A node is randomly selected as a $\mathrm{CH}$ and it is executed in such a way that each node becomes a cluster head once in an epoch. The node itself makes this decision. At the beginning, every node, choose a random number which is between 0 and 1 and then determines a threshold T(s). For the current round the node becomes a cluster head if the picked number is less than the threshold $\mathrm{T}(\mathrm{s})$, it is calculated as shown below:

$$
T(s)=\left\{\begin{array}{lr}
\frac{p}{1-p\left(r \cdot \bmod \frac{1}{p}\right)} & \text { if } \mathrm{s} \in G \\
0 & \text { otherwise }
\end{array}\right.
$$


Where $p$ is the probability of a node to become a cluster head, $r$ is the current round number and $G$ is the set of nodes that have not been cluster heads in the last $1 / p$ rounds.

\subsection{SEP}

Smaragdakis et al. proposed Stable Election Protocol (SEP) for the heterogeneous wireless sensor network which increases network's reliability [10]. It extends the LEACH protocol in assigning election probabilities of nodes to be cluster heads dependent on the initial energy of a node.

The Stable Election Protocol is based on the weighted election probability and it provides an improvement in the stable region of the clustering hierarchy process using the typical parameters of heterogeneity, which are the fraction of advanced nodes denoted as $\mathrm{m}$ and also the additional energy factor between the advanced and normal nodes denoted as $\alpha$. The weighted election probabilities for the normal and the advanced nodes are $P_{n r m}$ and $P_{a d v}$ respectively:

$$
\begin{aligned}
P_{n r m} & =\frac{P_{o p t}}{1+\alpha \cdot m} \\
P_{a d v} & =\frac{P_{o p t}}{1+\alpha \cdot m} \times(1+\alpha)
\end{aligned}
$$

The threshold for normal and advanced nodes is given below:

$$
\begin{gathered}
T\left(s_{n r m}\right)=\left\{\begin{array}{lr}
\frac{p_{n r m}}{1-p_{n r m} \cdot\left(r \bmod \frac{1}{p_{n r m}}\right)} & \text { if } s_{n r m} \in G^{\prime} \\
0 & \text { otherwise }
\end{array}\right. \\
T\left(s_{a d v}\right)=\left\{\begin{array}{lr}
\frac{p_{a d v}}{1-p_{a d v} \cdot\left(r \bmod \frac{1}{p_{a d v}}\right)} & \text { if } s_{a d v} \in G^{\prime} \\
0 & \text { otherwise }
\end{array}\right.
\end{gathered}
$$

Where $G^{\prime}$ and $G^{\prime \prime}$ are the set of normal and advanced nodes that have not become $\mathrm{CHs}$ in the last $1 / P_{n r m}$ and $1 / P_{a d v}$ round respectively.

\subsection{TSEP}

Javaid et al. proposed Threshold Sensitive Stable Election Protocol (TSEP) [12]. In the TSEP protocol, the cluster heads are selected on the basis of the threshold. This protocol considers three types of nodes that have different energy levels, called as the advance, intermediate and normal nodes. The initial energy for normal nodes is $E_{0}$, for advanced nodes, $E_{a d v}=E_{0}(1+\alpha)$ and for intermediate nodes is $E_{\text {int }}=E_{0}(1+\mu)$, where $\alpha$ and $\mu$ both are energy factor for advanced and intermediate nodes respectively and $\mu=\alpha / 2$. The probabilities to become normal, intermediate and advanced nodes are $P_{n r m}$, $P_{i n t}$ and $P_{a d v}$ respectively.

$$
\begin{gathered}
P_{n r m}=\frac{P_{o p t}}{1+m \cdot \alpha+b \cdot \mu} \\
P_{\text {int }}=\frac{P_{o p t}(1+\mu)}{1+m \cdot \alpha+b \cdot \mu}
\end{gathered}
$$

$$
P_{a d v}=\frac{P_{o p t}(1+\alpha)}{1+m \cdot \alpha+b \cdot \mu}
$$

To ensure that cluster head selection is done as assumed above, the threshold level is taken into consideration. The threshold for normal, intermediate and advanced nodes are given below:

$$
\begin{aligned}
& T_{n r m}=\left\{\begin{array}{lr}
\frac{p_{n r m}}{1-p_{n r m}\left(r \cdot \bmod \frac{1}{p_{n r m}}\right)} & \text { if } \mathbf{n}_{n r m} \in G^{\prime} \\
0 & \text { otherwise }
\end{array}\right. \\
& T_{\text {int }}=\left\{\begin{array}{lr}
\frac{p_{\text {int }}}{1-p_{\text {int }}\left(r \cdot \bmod \frac{1}{p_{\text {int }}}\right)} & \text { if } \mathbf{n}_{\text {int }} \in G^{\prime \prime} \\
0 & \text { otherwise }
\end{array}\right. \\
& T_{a d v}=\left\{\begin{array}{lr}
\frac{p_{a d v}}{1-p_{a d v}\left(r \cdot \bmod \frac{1}{p_{a d v}}\right)} & \text { if } \mathbf{n}_{a d v} \in G^{\prime \prime} \\
0 & \text { otherwise }
\end{array}\right.
\end{aligned}
$$

Where $\mathrm{G}^{\prime}, \mathrm{G}^{\prime \prime}$, and $\mathrm{G}^{\prime \prime \prime}$ are the set of normal, intermediate and advanced nodes that have not become $\mathrm{CHs}$ in the last $1 / P_{n r m}$, $1 / P_{i n t}$ and $1 / P_{a d v}$ round respectively. The TSEP protocol increases the stability, lifetime of the network because of three levels of heterogeneity and it also increases the throughput of the network. As TSEP is a reactive routing protocol, thus the nodes keep on sensing continuously, but the transmission is not done in a frequent manner.

\subsection{Cluster head election using Fuzzy Logic}

Gupta et al. proposed a clustering protocol using fuzzy logic which has input variables such as node's energy, concentration (number of nodes in the neighborhood) and centrality (how much node is at the center of the cluster) for cluster head selection [13]. However, it had increased transmission overhead. Cluster Head Election mechanism using Fuzzy Logic (CHEF) [7] proposed that the overhead of cluster head election may be highly reduced by using fuzzy logic with residual energy of node and the local distance (sum of distances between particular node and its neighbors within a specified radius) as fuzzy descriptors for cluster head selection at the node. The disadvantage of CHEF is that it did not consider inter-cluster communication cost for cluster head selection. Anno et. al. proposed a Cluster Head Election protocol that uses fuzzy logic with a distance of cluster centroid from BS, the residual energy of node and network traffic as inputs for cluster head selection [14]. Shen et al. [15] and applied fuzzy logic in heterogeneous sensor networks. Different fuzzy variables like concentration, centrality, energy, local distance, and distance from the base station were used for the fuzzy if-then rule. They examined that fuzzy logic can efficiently prolong the network lifetime.

\section{RADIO ENERGY DISSIPATION MODEL}

The radio energy dissipation model used in the proposed protocol is illustrated in Figure 1. We use equations which are given in [5] to compute the energy consumed for transmitting a $L$-bit packet from the transmitter to the receiver at a distance $d$ is defined as:

$E_{T x}(L, d)= \begin{cases}L \cdot E_{\text {elec }}+L \cdot \varepsilon_{f s} \cdot d^{2} & \text { if } \mathrm{d} \leq \mathrm{d}_{0} \\ L \cdot E_{\text {elec }}+L \cdot \varepsilon_{m p} \cdot d^{4} & \text { if } \mathrm{d}>\mathrm{d}_{0}\end{cases}$ 
where, $E_{\text {elec }}$ is the energy expended per bit to run the transmitter or receiver circuitry and $d_{0}$ is the threshold distance and is calculated as (13):

$d_{0}=\sqrt{\frac{\varepsilon_{f s}}{\varepsilon_{m p}}}$

The free space model $\left(\varepsilon_{f s}\right)$ and the multipath fading channel model $\left(\varepsilon_{m p}\right)$ are two different radio models which are used. The distance between the transmitter and the receiver is denoted as $d$. If $d$ is less than $d_{0}$ then the free space model is used, otherwise multipath fading channel model is used. $E_{R x}$ is the energy expended for receiving $L$ bits and is calculated as (14):

$E_{R x}(L)=L \cdot E_{\text {elec }}$

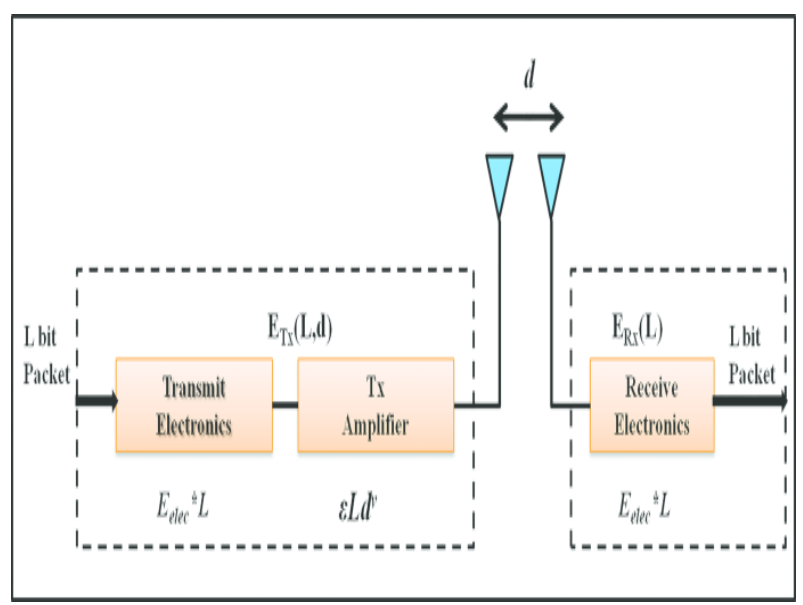

Fig 1: Radio Energy Dissipation Model

\section{FUZZY APPROACH}

Figure 2 shows the general Fuzzy Logic Controller (FLC) structure with Fuzzifier, Inference Engine, Fuzzy Rule Base (FRB) and Defuzzifier. The crisp input is fuzzified through the predefined membership functions. The Fuzzy Rule Base are a set of rules that are applied to the fuzzified input. The output of the inference engine is converted to crisp output by the process of defuzzification[16].

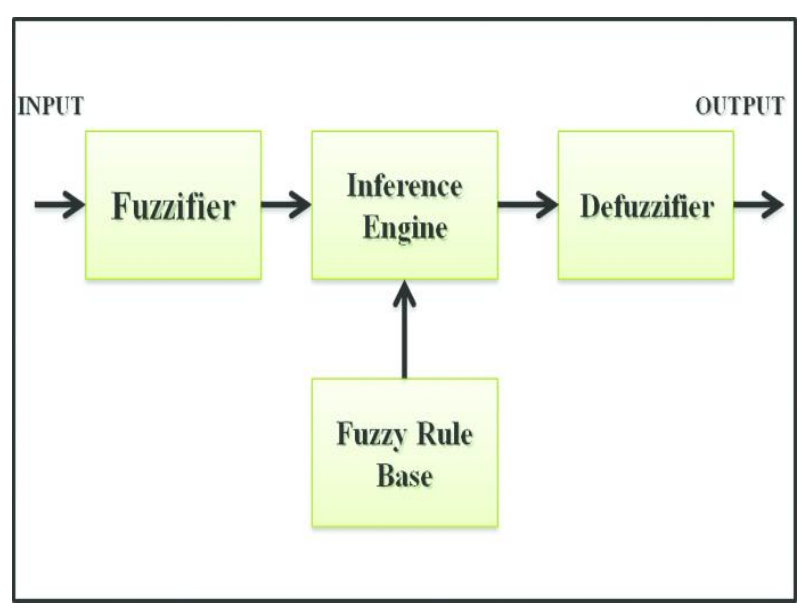

Fig 2: Fuzzy Logic Controller (FLC) Structure

\section{PROPOSED PROTOCOL}

In this section, we introduce our Enhanced Threshold Sensitive Stable Election Protocol using Fuzzy Logic (ETSSEPFL) for Wireless Sensor Networks.

\subsection{Network Model}

The Network model of this study is depicted in Figure 3. Let $n$ be the number of sensor nodes deployed randomly in a $\mathrm{M} \times \mathrm{M}$ area. The assumption for our model are as follows: All the deployed sensor nodes are stationary, the wireless sensor network consists nodes with different energy level i.e. heterogeneous sensor nodes, the Base Station (BS) is located at the center of the network area and the distance measurement is based on the wireless radio signal power.

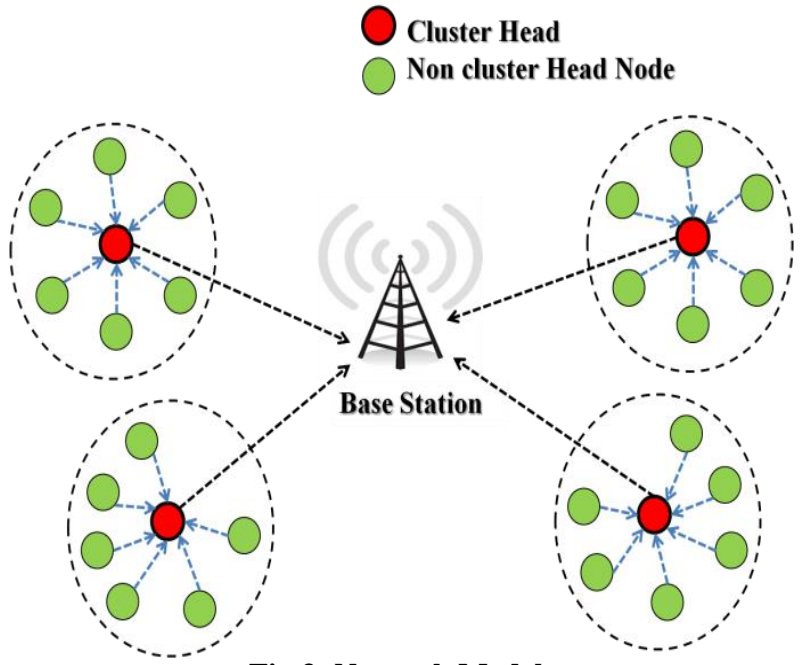

Fig 3: Network Model

\subsection{Fuzzy Inference System (FIS) for ETSSEPFL}

The Mamdani Model has been used for developing FIS for cluster head selection since it is intuitive, has widespread acceptance and is well suited to imprecise inputs [17]. The model of Fuzzy Interference System (FIS) for ETSSEPFL is depicted in Figure 4. The two input linguistic variables are Residual Energy and Distance to BS and one output variable is Probability.

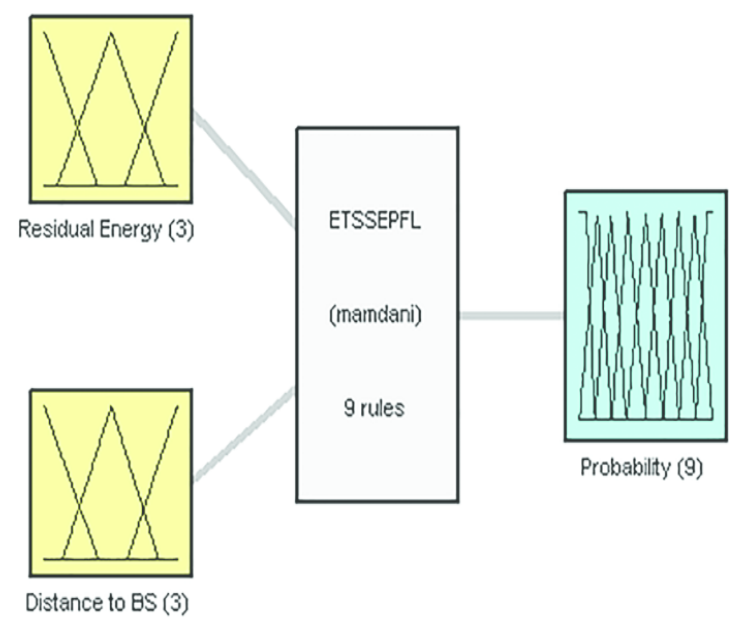

Fig 4: Proposed FIS Model for ETSSEPFL 


\subsection{FIS parameters and Rule Base}

The input linguistic variables used to represent the Residual Energy and Distance to BS is divided into three levels as follows:

Residual Energy $=\{$ Low, Medium, High $\}$

Distance to BS $=\{$ Close, Medium, Far $\}$

The output linguistic variable representing the Probability is divided into nine levels as follows:

Probability $=\{$ Very Small, Small, Rather Small, Small Medium, Medium, Very Medium, Small Large, Large, and Very Large \}

In proposed fuzzy system we use Triangular and Trapezoidal membership functions for the three linguistic variables since their degree can be easily determined [17]. The fuzzy sets are illustrated in Figure 5. With 2 input variables and 3 levels for each; there are $32=9$ possible combinations for rule base. To get the probability of becoming a $\mathrm{CH}$ we have used the fuzzy if-then rules that are defined with "and" operators among input variables and are shown in Table 1 . The aggregate of a fuzzy set includes a range of output values, and so must be defuzzified in order to resolve a single output value from the set. Centroid method is used for defuzzification.

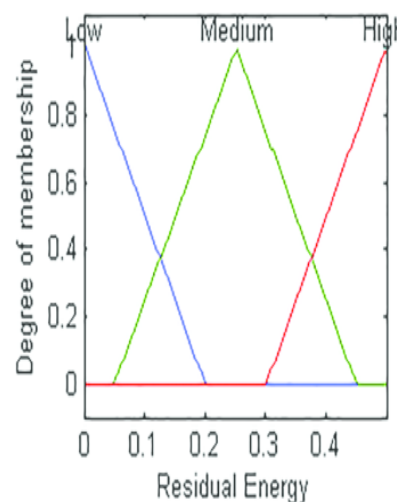

(a)

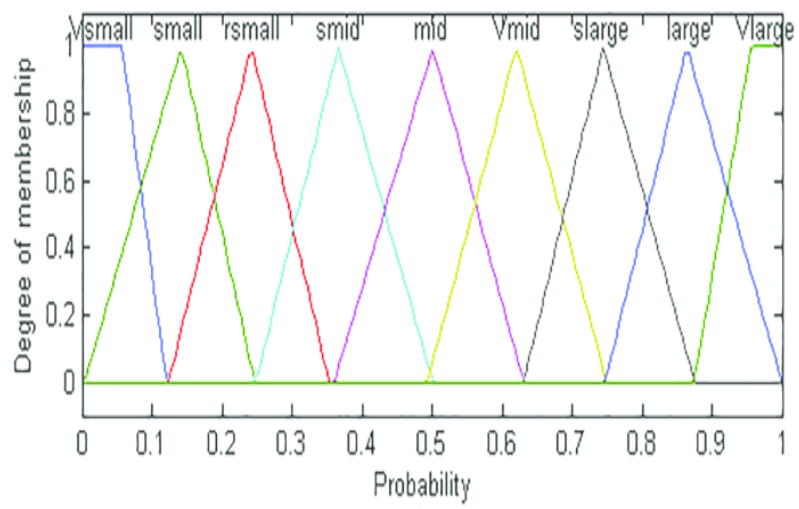

(c)

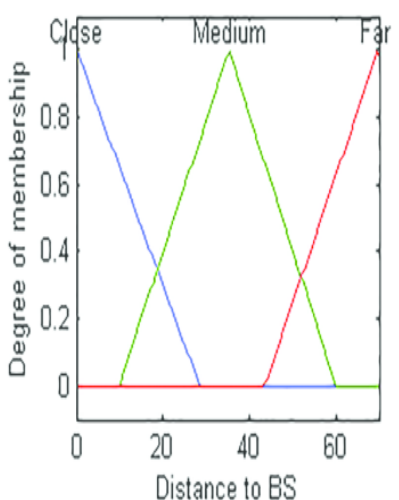

(b)
Fig 5: Fuzzy sets: (a) Membership function for Residual Energy, (b) Membership function for Distance to BS, (c) Membership function for Probability

The Table 1 shows that a sensor node that has a greater distance from the base station and less residual energy has the lowest possibility to be a $\mathrm{CH}$. On the other hand, a sensor node that has a lower distance from the base station and high residual energy has the highest probability to be a $\mathrm{CH}$.
Table 1. Fuzzy Rule Base

\begin{tabular}{|c|c|c|c|}
\hline Rules & $\begin{array}{c}\text { Residual } \\
\text { Energy }\end{array}$ & $\begin{array}{c}\text { Distance to } \\
\text { BS }\end{array}$ & $\begin{array}{c}\text { Probability to be } \\
\text { CH }\end{array}$ \\
\hline 1 & Low & Far & Very small \\
\hline 2 & Low & Medium & Small \\
\hline 3 & Low & Close & Rather small \\
\hline 4 & Medium & Far & Small medium \\
\hline 5 & Medium & Medium & Medium \\
\hline 6 & Medium & Close & Very medium \\
\hline 7 & High & Far & Small Large \\
\hline 8 & High & Medium & Large \\
\hline 9 & High & Close & Very large \\
\hline
\end{tabular}

Figure 6 depicts the flowchart for evaluating the Probability to be a cluster head using the Fuzzy Inference System (FIS) with two inputs that are Residual Energy and Distance to BS for ETSSEPFL protocol.

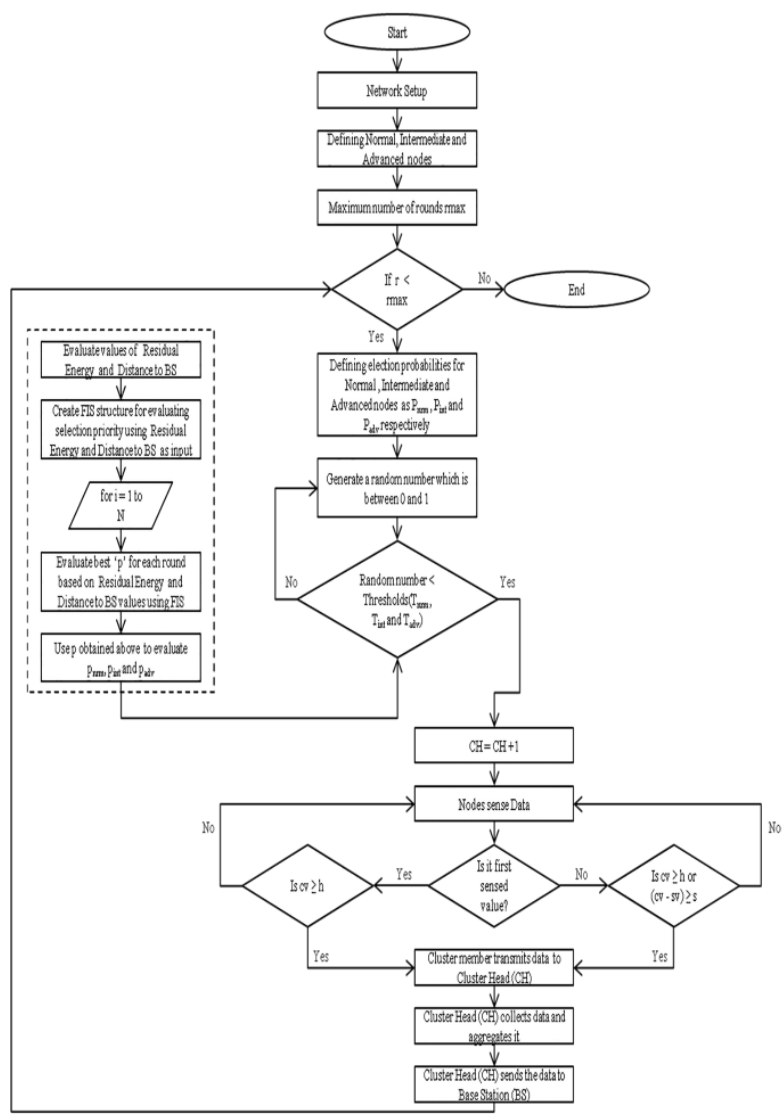

Fig 6: Flowchart of ETSSEPFL protocol

\section{SIMULATION AND ANALYSIS OF ETSSEPFL}

The Performance of ETSSEPFL is compared with protocol by Smaragdakis et al. and Javaid et al. Simulation parameters are shown in Table 2. 
Table 2. Simulation Parameters

\begin{tabular}{|c|c|c|}
\hline Description & Parameters & Value \\
\hline Network Size & $\mathrm{M} \times \mathrm{M}$ & $100 \times 100$ \\
\hline $\begin{array}{c}\text { Base Station } \\
\text { Location }\end{array}$ & BS & $(50,50)$ \\
\hline Number of Nodes & $\mathrm{n}$ & 100 \\
\hline $\begin{array}{l}\text { Initial Energy of } \\
\text { Nodes }\end{array}$ & $\mathrm{E}_{0}$ & $0.5 \mathrm{~J}$ \\
\hline $\begin{array}{l}\text { Energy dissipated } \\
\text { per bit }\end{array}$ & $\mathrm{E}_{\text {elec }}$ & $50 \mathrm{~nJ} / \mathrm{bit}$ \\
\hline $\begin{array}{c}\text { Transmit amplifier } \\
\text { if } \mathrm{d}_{\mathrm{toBS}} \leq \mathrm{d}_{0}\end{array}$ & $\varepsilon_{\mathrm{fs}}$ & $10 \mathrm{pJ} / \mathrm{bit} / \mathrm{m}^{2}$ \\
\hline $\begin{array}{c}\text { Transmit amplifier } \\
\text { if } \mathrm{d}_{\mathrm{toBS}}>\mathrm{d}_{0}\end{array}$ & $\varepsilon_{\mathrm{mp}}$ & $0.0013 \mathrm{pJ} / \mathrm{bit} / \mathrm{m}^{4}$ \\
\hline $\begin{array}{c}\text { Data aggregation } \\
\text { energy by } \mathrm{CH}\end{array}$ & $\mathrm{E}_{\mathrm{DA}}$ & 5nJ/bit/message \\
\hline Size of data packet & $\mathrm{K}$ & 4000bits \\
\hline $\begin{array}{c}\text { Proportion of } \\
\text { advanced nodes }\end{array}$ & $\mathrm{m}$ & 0.1 \\
\hline $\begin{array}{l}\text { Energy factor for } \\
\text { Advanced nodes }\end{array}$ & $\alpha$ & 1 \\
\hline
\end{tabular}

Based on the above network features and parameters, the protocol was implemented and examined using MATLAB. Figure 7 shows the number of alive nodes for SEP, TSEP and ETSSEPFL protocols and it is clear that ETSSEPFL outperforms the SEP and TSEP protocols. Table 3 also clarify this as in SEP and TSEP first node died at 986th and 2687th round respectively while in ETSSEPFL first node died at 3211 th round. Hence the stability region of the network is significantly increased compared to SEP and TSEP protocols. Similarly, 50\% nodes died at the 1300th round, 3658th round and 4432nd round for SEP, TSEP, and ETSSEPFL respectively. The network is working for a longer period of time because the last node died at the 7002 nd round in ETSSEPFL while in SEP and TSEP it died at 2466th and the 5515th round respectively. When compared with SEP and TSEP protocols the proposed protocol remarkably improves the overall network lifetime.

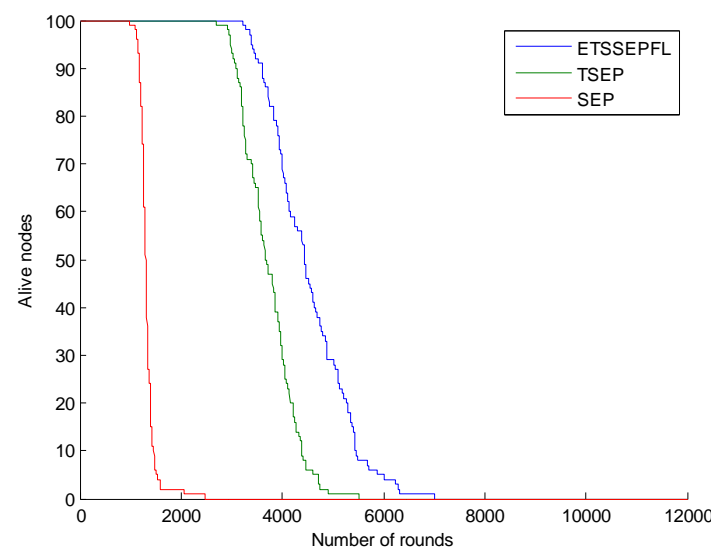

Fig 7: Number of Alive nodes per round

Table 3. Comparison of dead nodes

\begin{tabular}{|c|c|c|c|}
\hline \multirow{2}{*}{ Nodes } & \multicolumn{3}{|c|}{ Number of rounds } \\
\cline { 2 - 4 } & SEP & TSEP & ETSSEPFL \\
\hline $1 \%$ & 986 & 2687 & 3211 \\
\hline $50 \%$ & 1300 & 3658 & 4432 \\
\hline $100 \%$ & 2466 & 5515 & 7002 \\
\hline
\end{tabular}

Figure 8 represents the number of packets sent to the BS in each round and it clearly specifies that throughput of ETSSEPFL is far better than SEP and TSEP.

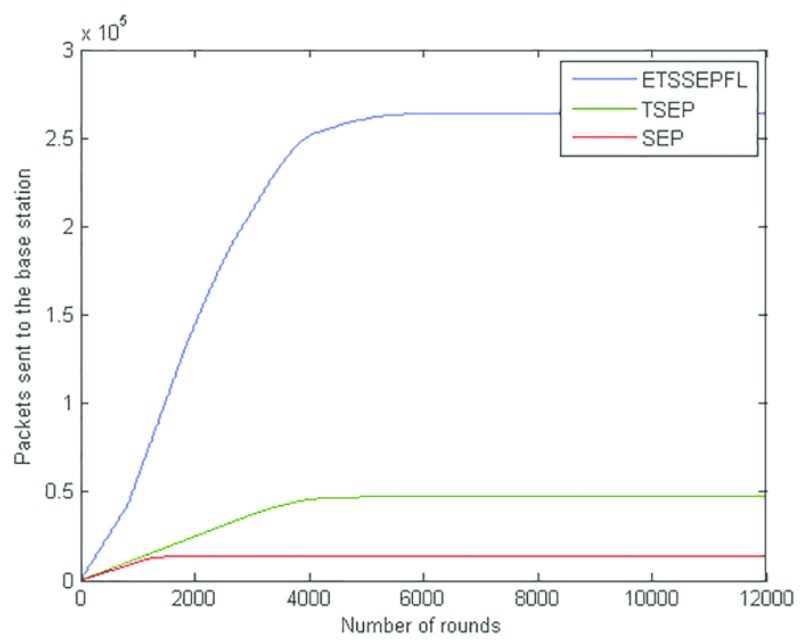

Fig 8: Throughput of the protocols

Figure 9 shows the surface plot of the two input variables residual energy and distance to base station and the output variable probability of $\mathrm{CH}$. As the energy level increased and the distance to base station decreased, the node is more probable to elect as a $\mathrm{CH}$. 


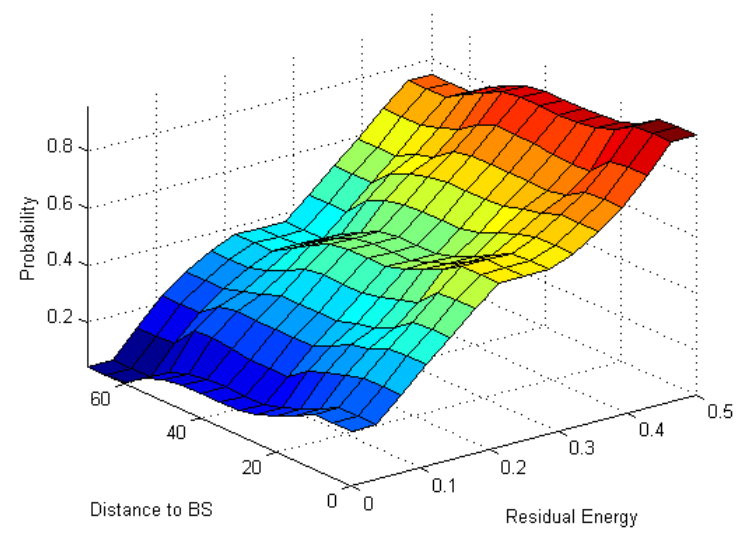

Fig 9: Surface plot for Residual energy and Distance to base station

Figure 10 describes the stability period of SEP, TSEP, and ETSSEPFL. By comparing these results it is found that the stability period of SEP is at round 986th while for TSEP and ETSSEPFL the stability period it is at round 2687th and 3211th respectively. Therefore ETSSEPFL outperformed both SEP and TSEP in terms of stability period.

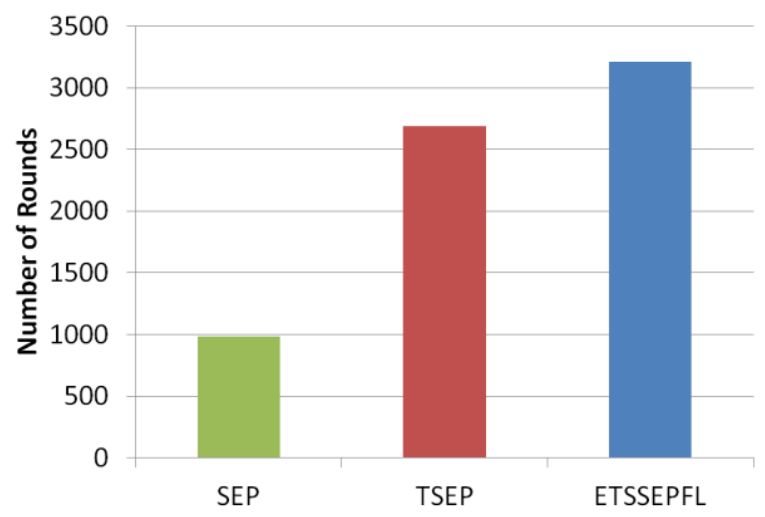

Fig 10: Stability period of protocols in rounds

Figure 11 shows the instability period of the protocols, SEP instability period is at round 1480th while for TSEP and ETSSEPFL it is at round 2828th and 3791st respectively. It clearly exhibits that instability period of ETSSEPFL is, far better than SEP and TSEP.

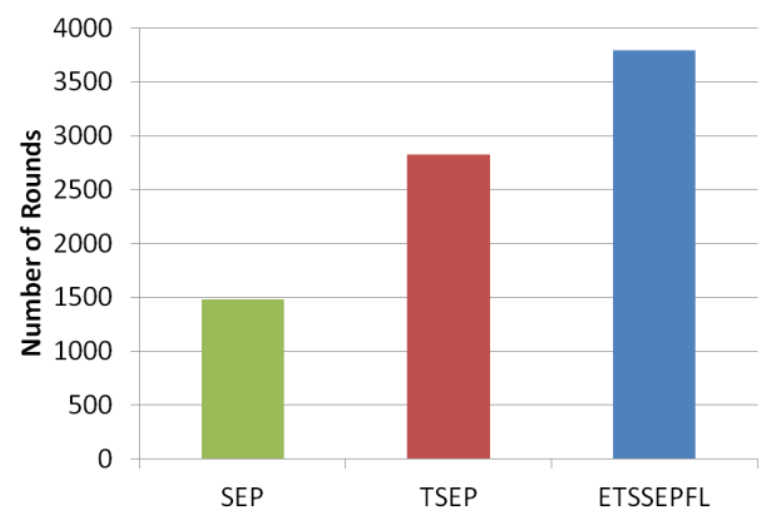

Fig 11: Instability period of protocols in rounds

Figure 12 shows the lifespan of SEP, TSEP, and ETSSEPFL. In the SEP protocol the last node dies at 2466th round while for TSEP and ETSSEPFL the last node dies at the 5515th and 7002nd rounds respectively. This indicates that the lifetime, of ETSSEPFL, is better than the other two protocols.

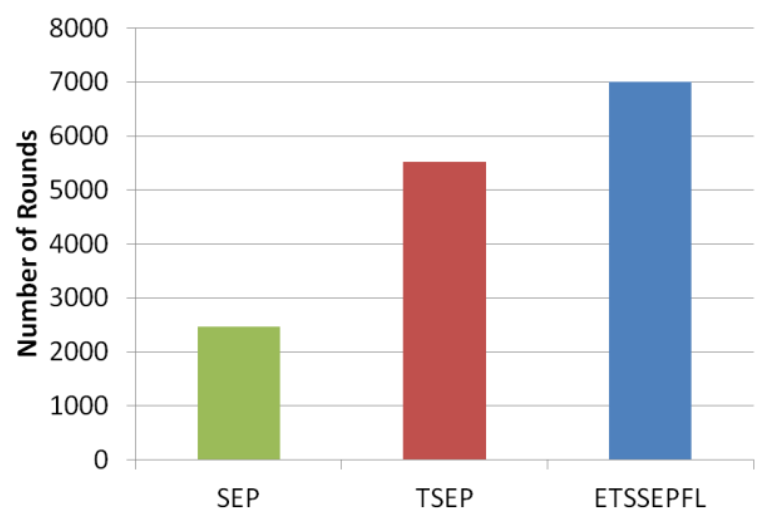

Fig 12: Lifespan versus number of rounds

Figure 13 clearly specify that throughput of ETSSEPFL is far better than SEP and TSEP. The number of packets sent to the base station in SEP, TSEP, and ETSSEPFL is 13,530, 47,280 and 2,64,400 respectively.

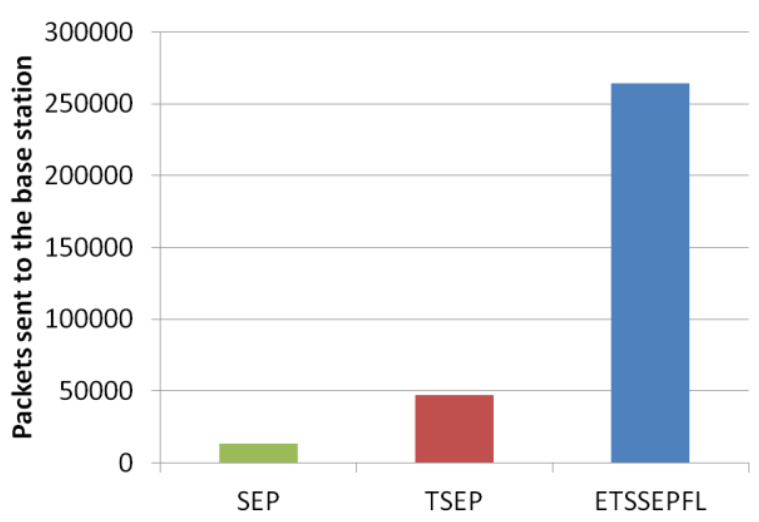

Fig 13: Throughput of the protocols

Figure 14 describes the stability versus instability period of SEP, TSEP, and ETSSEPFL.

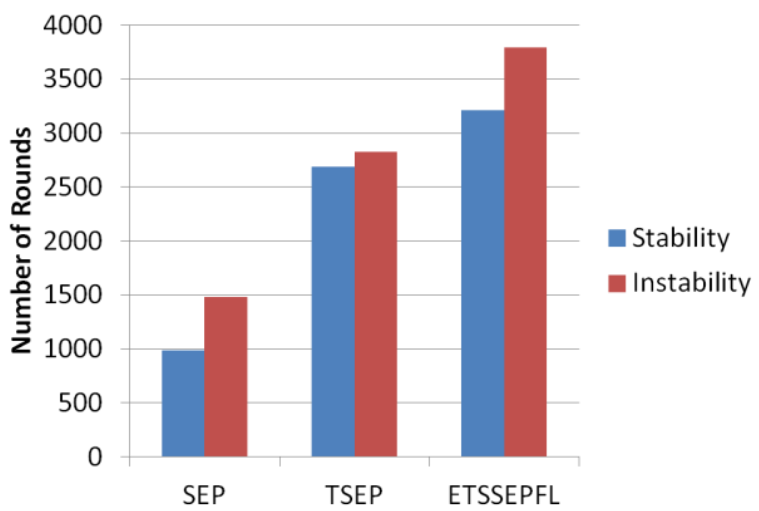

Fig 14: Stability versus Instability periods of protocols

ETSSEPFL clearly outperforms both SEP and TSEP by enlarging the stability period, lifetime and throughput. The performance analysis of ETSSEPFL against SEP and TSEP is shown in Table 4. 
Table 4. Comparison table of TSEP and ETSSEPFL

\begin{tabular}{|c|c|c|c|c|}
\hline Protocol & $\begin{array}{c}\text { Stabilit } \\
\mathbf{y} \\
\text { Period }\end{array}$ & $\begin{array}{c}\text { Instabilit } \\
\mathbf{y} \text { period }\end{array}$ & $\begin{array}{c}\text { Lifetim } \\
\mathbf{e}\end{array}$ & $\begin{array}{c}\text { Throughp } \\
\mathbf{u t}\end{array}$ \\
\hline SEP & 986 & 1480 & 2466 & 13,530 \\
\hline TSEP & 2687 & 2828 & 5515 & 47,280 \\
\hline $\begin{array}{c}\text { ETSSEPF } \\
\text { L }\end{array}$ & 3211 & 3791 & 7002 & $2,64,400$ \\
\hline
\end{tabular}

\section{CONCLUSION}

In this paper, a novel fuzzy model for energy-optimized routing in heterogeneous wireless sensor network called ETSSEPFL protocol is presented and compared with SEP and TSEP. The simulation results have demonstrated the reliability and efficiency of this approach. ETSSEPFL achieves longer lifetime, increased stability and throughput than existing SEP and TSEP. This is because residual energy and distance to the base station are considered in selecting CHs. It was observed that ETSSEPFL outperforms existing SEP and TSEP protocols in terms of stability period, network lifetime and throughput and builds a more stable routing environment. Moreover, as the fuzzy approach is soft it can be easily tuned for different network and node conditions simply by changing shapes of the fuzzy sets. In this paper, the proposed ETSSEPFL protocol is designed for the heterogeneous wireless sensor network that has fixed base station or sink. In future, it can be extended using some additional fuzzy variables and for handling mobile base station.

\section{REFERENCES}

[1] I. Akyildiz, W. Su, Y. Sankarasubramaniam, and E. Cayirci, "A survey on sensor networks," IEEE Communications Magazine, vol. 40, no. 8, pp. 102-114, August 2002.

[2] I.F. Akyildiz, W.J. Su, Y. Sankarasubramaniam, E. Cayirci, "Wireless sensor networks: a survey", Computer Networks 38, 393-422, 2002.

[3] J.N. Al-Karaki, A.E. Kamal, "Routing techniques in wireless sensor networks: a survey", IEEE Wireless Communications, 2004.

[4] S. Gajjar, M. Sarkar and K. S. Dasgupta, "Cluster Head Selection Protocol using Fuzzy Logic for Wireless Sensor Networks", International Journal of Computer Applications, 38-43,2014.

[5] W. R. Heinzelman, A. Chandrakasan, and H. Balakrishnan, "Energy-efficient communication protocol for wireless microsensor networks", System Sciences. In Proceedings of the 33rd annual Hawaii international conference, pp. 4-7, 2000.

[6] O. Younis, S. Fahmy, "Heed: a hybrid, energy- efficient distributed clustering approach for Ad hoc sensor networks," IEEE Trans on mobile computing, 3(4), pp.660-669, 2004.

[7] J. M. Kim, S.H. Park, Y. J. Han, and T.M. Chung, "CHEF: Cluster Head Election mechanism using Fuzzy logic in Wireless Sensor Networks," Advanced Communication Technology, ICACT 2008, Vol. 1, pp. $654-6592008$

[8] D. Kumar, T. C. Aseri, and R. B. Patel, "EEHC: Energy efficient heterogeneous clustered scheme for wireless sensor networks", Computer Communications, 32(4), 662-667, 2009.

[9] P. Saini, A. K. Sharma, "Energy efficient scheme for clustering protocol prolonging the lifetime of heterogeneous wireless sensor networks", International Journal of Computer Applications, 6(2), 30-36, 2010.

[10] G. Smaragdakis, I. Matta, and A. Bestavros, "SEP: A stable election protocol for clustered heterogeneous wireless sensor networks," Second International Workshop on Sensor and Actor Network Protocols and Applications, July 2004.

[11] L.Qing, Q. X.Zhu, and M. W.Wang, "A distributed Energy-Efficient Clustering Algorithm for Heterogeneous Wireless Sensor Networks," Journal of Software, Vol.17, No. 3, pp. 481-489, 2006.

[12] A. Kashaf, N. Javaid, Z. A . Khan, and I. A. Khan, "TSEP: Threshold-sensitive stable election protocol for WSNs", In 10th international conference on frontiers of information technology (FIT) (Vol.164, no. 168, pp. 1719) December 2012.

[13] I. Gupta, D. Riordan, S. Sampalli, "Cluster-head election using fuzzy logic for wireless sensor networks", in Proc. of Annual Communication Networks and Services Research Conference, pp. 255-260, 2005.

[14] J. Anno, L. Barolli, F. Xhafa, and A. Durresi, "A Cluster Head Selection Method for Wireless Sensor Networks Based on Fuzzy Logic", Proc. of IEEE TENCON, pp. 14, 2007.

[15] Y. Shen, H. Ju, "Energy-Efficient Cluster-head Selection based on a fuzzy expert system in wireless sensor networks", IEEE/ACM International Conference on Green Computing and Communications(GreenCom), Chengdu, China, August 4-5, 2011.

[16] H. Natarajan and S. Selvaraj, "A Fuzzy Based Predictive Cluster Head Selection Scheme for Wireless Sensor Networks", Proceeding of the 8th International Conference on Sensing Technology, Sep. 2-4, Liverpool, UK, 2014

[17] J S R Jang, C T Sun and E Mizutani, "Neuro-fuzzy and soft computing A Computational approach to learning and machine intelligence", Prentice Hall Publications, 2003. 